\title{
Accreditation program for gastrointestinal endoscopes reprocessing in Italy: An on-site survey
}

\section{다 $(9)$}

\section{Authors}

Giancarlo Spinzi ${ }^{1}$, Angelo Milano², Piero Brosolo ${ }^{3}$, Paola Da Massa Carrara ${ }^{4}$, Maurizio Labardi ${ }^{5}$, Alberto Merighi ${ }^{6}$, Luisa Riccardi ${ }^{7}$, Francesco Torresan ${ }^{8}$, Maurizio Capelli ${ }^{9}$

Institutions

1 Gasastroenterology and Endoscopy Department, Valduce Hospital, Como - Italy

2 Gastroenterology and Endoscopy Department, Chieti University, Chieti - Italy

3 Gastroenterology and Endoscopy Department, Pordenone Hospital, Pordenone - Italy

4 Gastroenterology and Endoscopy Department, Pistoia Hospital, Pistoia - Italy

5 Gastroenterology and Endoscopy Department, Firenze Hospital, Firenze - Italy

6 Gastroenterology and Endoscopy Department, Ferrara Hospital, Ferrara - Italy

7 Gastroenterology and Endoscopy Department, Perugia Hospital, Perugia - Italy

8 Gastroenterology and Endoscopy Department, Bologna University, Bologna - Italy

9 Kiwa-Cermet Statistical Department, Bologna-Italy

submitted 26.12.2020

accepted after revision $\quad 10.6 .2021$

Bibliography

Endosc Int Open 2021; 09: E1627-E1632

DOI 10.1055/a-1535-1238

ISSN 2364-3722

(C) 2021. The Author(s).

This is an open access article published by Thieme under the terms of the Creative Commons Attribution-NonDerivative-NonCommercial License, permitting copying and reproduction so long as the original work is given appropriate credit. Contents may not be used for commercial purposes, or adapted, remixed, transformed or built upon. (https://creativecommons.org/licenses/by-nc-nd/4.0/)

Georg Thieme Verlag KG, Rüdigerstraße 14,

70469 Stuttgart, Germany

Corresponding author

Giancarlo Spinzi, MD, Valduce Hospital, Via Dante 11, 22100

Como, Italy
Fax: +39031308047

gispinz@tin.it

$\circledast$ Supplementary material is available under https://doi.org/10.1055/a-1535-1238

\section{ABSTRACT}

Background and study aims Endoscope reprocessing has been associated with a variable failure rate. Our aim was to present an overview on current practices for reprocessing in Italian facilities and discuss the principle critical points.

Methods In 2014 the Italian Society for Digestive Diseases implemented an accreditation program in collaboration with an independent organization for certification and with the Italian Association for Endoscopy Technical Operators. During a 1-day site visit of the endoscopy center, two endoscopists, one nurse, and the representative of the certification body evaluated the endoscope reprocessing.

Results As of July 1, 2020, 28 endoscopy centers had been accredited. Ten centers are completing the measures to correct deficiencies found at the visit. Three centers withdrew from the program. The accreditation program has found variations between the various centers, confirming the poor compliance with guidelines. Major deviations from the standards, established by the model before the site visit according to national and international guidelines, concerned instrument cleaning (44.7\% of the centers), instrument storage $(23.7 \%)$, and microbiological tests (31.6\%).

Conclusions Our overview demonstrated the lack of many reprocessing phases, which are important to prevent endoscopy-associated infections. Accreditation can achieve a transformation in quality and safety of reprocessing with the Italian centrally-led approach.

\section{Background}

Gastrointestinal endoscopy is widely performed for diagnosis and treatment of patients with gastrointestinal diseases and it is also useful in healthy people who require clinical examinations or checkups. Endoscopy has changed significantly over the last 30 years as technological developments have established a great variety of diagnostic and therapeutic options. 
Flexible endoscopes are reusable medical devices with multiple lumens and narrow channels. By definition, gastrointestinal endoscopes are semi-critical medical equipment requiring major quality assurance for disinfection [1].

Because endoscopic devices are temperature-sensitive, lowtemperature chemical methods, such as liquid chemical germicide, must be used rather than steam sterilization. However, these instruments are difficult to clean and disinfect and easy to damage because of their complex design. Inadequate reprocessing of endoscopes or endoscopic accessories may result in infection outbreaks. In addition, the ability of bacteria to form a biofilm in the endoscopic channels, especially when the channels become damaged, can contribute to failure of the decontamination process.

Although the incidence of iatrogenic infection during gastrointestinal endoscopy was only about 1 in 1.8 million procedures in the United States from 1988 to 1992 [2], outbreaks of bacterial/viral infections, complicated by the contamination of endoscopes and washer-disinfector instruments, were also individually reported [3].

The US Food and Drug administration (FDA) has received notification of 142 cases of patient infections or exposure from reprocessed duodenoscopes since 2010 [4].

However, the true risk of transmission during endoscopy may go unrecognized because of technically inadequate surveillance, no surveillance at all, low frequency, or the absence of clinical symptoms.

In 2015, the FDA issued a safety alert and ascertained concerns about an association between multidrug-resistant bacterial infections and duodenoscopic investigations [5].

Colonoscopy-related infections and complications have been reported in multiple studies, although at lower rates compared with endoscopic retrograde cholangiopancreatography (ERCP) [6]. Colonoscopy infection rates are estimated at 3.7 and 1.6 per 1000 procedures with high costs per hospitalization [7].

These infections have occurred in American as well as European centers, and have also been widely reported by the media in view of their clinical impact [8].

Since 1994, the Guideline Committee of the European Society of Gastrointestinal Endoscopy (ESGE) and the European Society of Gastrointestinal Nurses and Associates (ESGENA) have developed a number of guidelines and position statements focused on hygiene and infection control in endoscopy [9].

In 2019, a consensus document on the competencies required by healthcare staff to deliver adult endoscopy services throughout the UK was prepared by nursing representatives from the British Society of Gastroenterology Nurses Associates, the Royal College of Nursing and the Joint Advisory Group for Gastrointestinal Endoscopy [10]. This consensus document was created in partnership with the manufacturers of endoscopes and decontamination equipment, Direct Observation of Procedural Skill Forms, specifically on decontamination for technicians of the procedure. This document is available on the contamination page of the British Society of Gastroenterology website [11].
Many other international societies for gastrointestinal endoscopy have also issued guidelines for endoscopy reprocessing. These guidelines are constantly revised with the introduction of new disinfectants and devices.

In 2014, the Italian Society for Digestive Endoscopy (SIED) designed and implemented an accreditation program for endoscopy services, in collaboration with an independent international certification organization and the Italian Association for Endoscopy Technical Operators (ANOTE) [12].

In recent years, endoscopy units from a range of private and public institutions have been voluntarily surveyed by means of a dedicated questionnaire and a 1-day on-site visit.

In light of raised awareness about the need for reprocessing of all types of endoscopes to prevent transmission of infections, we present an overview of the current accreditation program for gastrointestinal endoscope reprocessing in Italian facilities. In addition, we will discuss critical issues found during endoscope reprocessing.

\section{Methods}

In 2014, SIED implemented an accreditation program in collaboration with KIWA CERMET ITALIA, an independent international organization for certification with a section specializing in healthcare, along with the Italian Association for Endoscopy Technical Operators. A team of eight endoscopists selected from different institutions and with at least 10 years' experience by the SIED council was given the task of drafting professional and service standards according to Italian [13] and international guidelines $[9,11]$ using a standardized system. For each center that voluntarily requests accreditation, a self-assessment checklist is provided as a tool for evaluating how closely they comply with the standards established by the model, before an on-site visit. The site visit applied by the centers lasts 1 day and is carried out by two expert endoscopists: the representative of the certification body -KIWA CERMET ITALIA - who ensures the fairness of procedures and a professional nurse nominated by ANOTE.

During the site visits to the endoscopy centers, the endoscopists and the nurse evaluate the endoscopic reports in a multidisciplinary approach, looking at the route taken as a patient is moved through the endoscopy unit, the nursing records, instrument reprocessing, and the technologies available (Appen$\operatorname{dix}$ A).

As the efficacy of endoscope reprocessing depends on the staff's comprehensive knowledge of the construction and function of the endoscope, during the site visit, the nurse checks the availability of detailed protocols describing the different phases of reprocessing. The nurse specifically checks four different phases of reprocessing: bedside cleaning, manual cleaning at the reprocessing area (including leak testing and brushing of endoscope channels), cleaning and disinfection, drying, and storage. Irrespective of the size and design of the reprocessing area, the nurse also examines personal protective equipment, adequate equipment for manual cleaning steps (e.g. brushes, cleaning adapters, endoscopy leak test units), appro- 
priate storage of process chemicals, compressed air for drying, documentation, and traceability equipment.

The nurse then verifies that the documentation for the reprocessing procedure includes the patient's name, the endoscope identification, the whole reprocessing cycle, and identification of the staff member involved in reprocessing. Microbial culture examination of the endoscope and its accessories is then certified.

At the end of the day's visit, the findings are presented and discussed during a meeting with the center's endoscopists, some of the nursing staff, and the representative of the medical and/or general management, the latter to approve relevant actions.

Service standards have been approved by SIED and ANOTE boards and are available to all SIED members and can be freely consulted on the site www.sied.it (in Appendix A only gastrointestinal reprocessing standards are reported).

Any non-compliance with SIED standards that comes to light during a site visit must be corrected by the endoscopy unit in the time assigned for the accreditation procedure. The team then checks whether the unit has implemented all the corrective measures required. The SIED accreditation program requires renewal every 2 years after the first site visit, in which all critical issues are resolved, in order to obtain accreditation.

\section{Statistical analysis}

To analyze the differences between the criteria reported in the structure's self-assessment as being "not met" and those found on the on-site visit, we used a non-parametric test for paired samples. A two-sided $P \leq 0.05$ was considered statistically significant.

\section{Results}

From the beginning of the SIED accreditation scheme in 2014 until June 2020, 41 site visits occurred. All endoscopy services visited were centers of reference that carry out more than 8000 exams per year. As of July 1, 2020, 28 endoscopy centers had been accredited. Ten centers were not accredited because they had not completed the measures to correct points raised at the visit. Three centers withdrew from the SIED-ANOTE program after the first site visit.

In the pre-site visit self-assessment, 41 centers reported the presence of 21 critical issues with respect to SIED standards. In the same centers, the critical points detected by the members of the SIED and ANOTE team were $92(P=0.008)$ ( $\triangleright$ Fig. 1). In seven centers no deviations from the program standards were noted. In $>$ Table 1 the critical points found in 31 centers for the reprocessing procedure are reported in detail.

Thorough manual cleaning with detergent was the most common deviation from guidelines, which was reported in 33 of 41 centers (80.5\%). Microbiological surveillance of a proportion of the department's endoscopes, with the requirements that all endoscopes used in the unit are tested at least once a year, was not correctly carried out in 13 of 41 centers (31.7\%). Storage in a controlled environment for the prevention of any secondary contamination was not considered adequate in 11

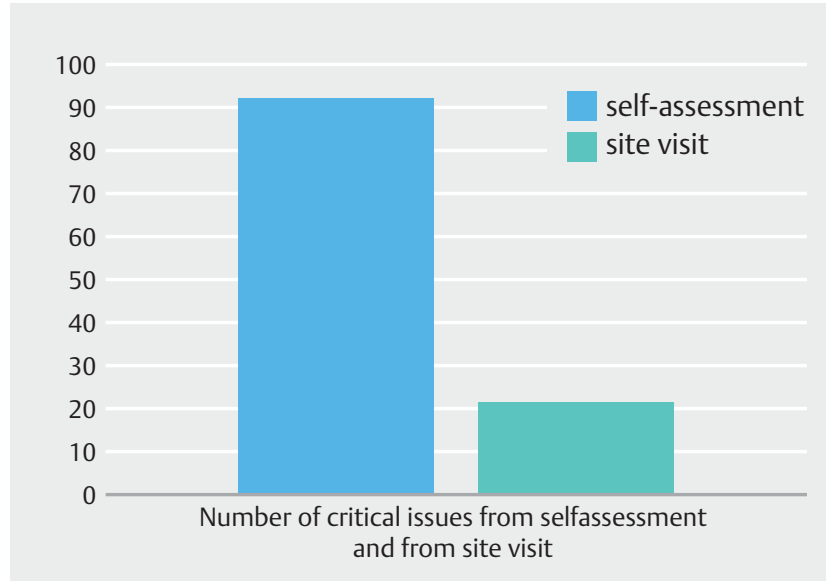

- Fig. 1 Number of critical issues from self-assessment and from site visit.

of 41 endoscopy units (26.8\%). Transport in closed containers, clearly marked as contaminated equipment in order to avoid contamination of the environment as well as third parties, was found insufficient in 10 of 41 centers (24.4\%). Reprocessing did not follow the officially required endoscopy reprocessing training program, nor was there regular practice and periodical updated training given to maintain competency in six of 41 centers (14.6\%). In six of 41 endoscopy services (14.6\%) nurses and allied healthcare professionals were not fully aware of their roles and responsibilities with regard to legislation concerning medicine management, professional accountability and responsibilities in the delegation of tasks. A complete reprocessing documentation was not considered adequate in five of 41 centers (12.2\%). Thorough drying of endoscope surfaces and channels to prevent any growth of waterborne microorganisms was wrongly performed in three of 41 endoscopy units (7.3\%).

Thirteen centers renewed their accreditation after two years, as scheduled. No critical issues were found in three of 13 centers. During the first site visit, we found 27 critical issues; 23 were found during the renewal site visit $(P=0.388)$ ( $($ Fig. 2 ). The results of the renewal accreditations are reported in $>\mathbf{T a}$ ble 2.

\section{Discussion}

The SIED-ANOTE accreditation program has found variations in the methods of reprocessing endoscopes in the centers which confirm their poor compliance with the numerous guidelines issued by many scientific societies [9-15]. This variability and poor adherence to guidelines has already been reported in the literature and is based on various issues. In the work of Thaler AM et al. only $53 \%$ of the 249 US hospitals considered performed surveillance microbiological culturing. Furthermore, forced air drying after reprocessing was used by $47.8 \%$ of centers [16]. Moreover, an international survey on current endoscopic reprocessing identified a large variation in practices: $41 \%$ of 165 services used adenosine triphosphate as an assessment method despite the fact that this technique has not yet 
- Table 1 Critical issues resulting from self-assessment and from the site visit.

\begin{tabular}{|l|l|l|}
\hline Specific reprocessing area & $\begin{array}{l}\text { No. of critical issues resulting from } \\
\text { self-assessment }\end{array}$ & $\begin{array}{l}\text { No. of critical issues resulting from } \\
\text { the site visit }\end{array}$ \\
\hline Standards of professional conduct & 0 & 6 \\
\hline Operator skills & 1 & 6 \\
\hline Compliance with reprocessing phases & 1 & 5 \\
\hline Predetersion & 0 & 0 \\
\hline Cleansing & 1 & 33 \\
\hline Disinfection & 0 & 0 \\
\hline Drying & 0 & 3 \\
\hline Storage & 6 & 11 \\
\hline Transport of contaminated equipment & 3 & 10 \\
\hline Microbiological tests & 6 & 13 \\
\hline Complete reprocessing documentation & 3 & 5 \\
\hline Total & 21 & 92 \\
\hline
\end{tabular}

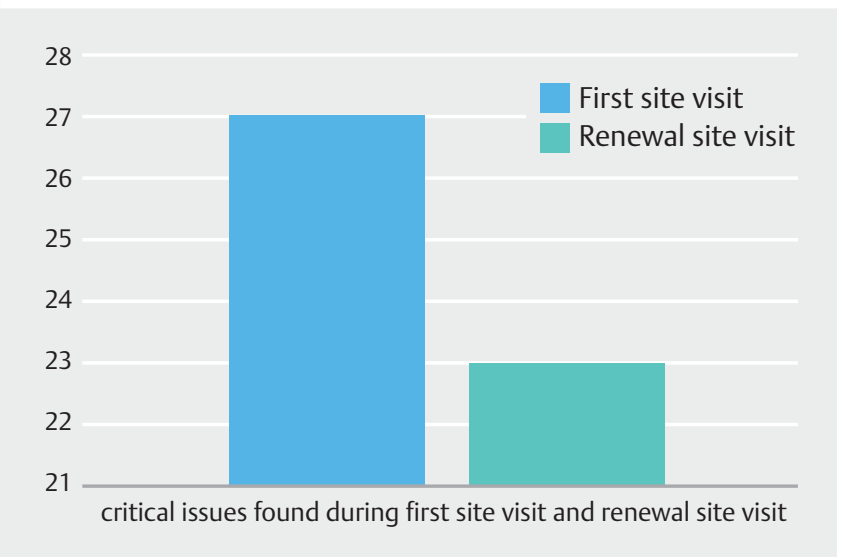

- Fig. 2 Critical issues found during first site visit and renewal site visit.

been recommended in guidelines, $33 \%$ of participating facilities reported their staff was still untrained, and only $57 \%$ of the respondents carried out the drying process routinely [17].

In our study, using direct surveys carried out by experienced medical and nursing staff, the major deviations from the guidelines concern manual instrument cleaning (33 of 41 centers: $80.5 \%$ ), instrument storage (11 of 41 centers: $26.8 \%$ ), the lack or failure to carry out microbiological tests (13 of 41 centers: $31.7 \%)$, incorrect transport in closed containers in 10 of 41 (24,4\%).

Moreover, we also found insufficient complete documentation in seven of 41 centers (17.1\%), insufficient operator skills and training in six of 41 centers (14.6\%), and incorrect drying of instruments in three of 41 centers (7.3\%).

In a recent review aimed at providing an update on endoscopy-associated infection, and the factors contributing to their occurrence, manual cleaning, which is crucial to prevent biofilm formation, was identified as inadequate in four of the revised articles [18]. Cleaning is the most important step in reprocessing. Bedside cleaning and the manual cleaning step with flushing and brushing of the entire channel systems are essential for the removal of debris, blood and body fluids. Biofilm formation is possible if any of the steps have not been carried out correctly. As some Gram-negative bacteria can undergo cell division every 20 to 30 minutes, it is essential to complete all reprocessing steps quickly, before bacterial growth and debris begin to dry on surfaces. Some national guidelines recommend performance of all manual reprocessing steps within 30 minutes after completion of the patient examination [9].

Microbial culture examination of the endoscopes and its accessories is recommended to be performed periodically to control the quality of the reprocessing procedure, and in the event of suspected endoscope-mediated infection, a microbial culture study is mandatory [19]. However, specific conditions and principles concerning the examination interval and culture methods have not yet been established. To date, cost-effectiveness analysis of microbial culture studies is limited. Some queries about the interpretation of microbial culture results and the approach to take for culture-positive devices are still not sufficiently answered. In a recent international survey from 39 countries, a large disparity in the use of microbial cultures was observed [17]. Microbial cultures were used twice a year in $25 \%$ of the centers. The final rinse water test was assessed monthly in only $22 \%$ of the participating endoscopy units.

Transportation of a contaminated endoscope in a sealed container to the disinfection area had the lowest compliance rate $(56.0 \%)$ in a recent study from Korea [20]. The same study compared compliance rates for contaminated endoscope transport between hospitals with $>100$ vs $<100$ average daily endoscopic examinations, and found no significant difference in compliance. The compliance rates regarding the transport 
Table 2 Critical issues

\begin{tabular}{|l|c|c|}
\hline Specific reprocessing area & No. of critical issues after first site visit & No. of critical issues after renewal visit \\
\hline Standards of professional conduct & 2 & 1 \\
\hline Operator skills & 2 & 0 \\
\hline Compliance with reprocessing phases & 0 & 1 \\
\hline Predetersion & 0 & 1 \\
\hline Cleansing & 10 & 7 \\
\hline Disinfection & 0 & 0 \\
\hline Drying & 0 & 1 \\
\hline Storage & 4 & 2 \\
\hline Transport of contaminated equipment & 2 & 2 \\
\hline Microbiological tests & 5 & 6 \\
\hline Complete reprocessing documentation & 2 & 2 \\
\hline Total & 27 & 23 \\
\hline
\end{tabular}

of contaminated endoscopes in the United States and Portugal are $26 \%$ and $44 \%$, respectively [21,22].

The complete reprocessing cycle should be documented, including the names of the persons undertaking each step and the reprocessing record should be reported in the patient's files [9]. However, a lack of complete documentation was observed in $7 / 41$ centers $(17.1 \%)$ in our on-site study.

Guidelines recommend, after reprocessing, to flush each endoscope channel with compressed air and $70 \%$ to $90 \%$ ethyl or isopropyl alcohol to facilitate drying [9]. As moisture breeds and encourages microbial bacteria such as Pseudomonas aeruginosa to multiply, drying is a crucial procedure [9]. Moreover, alcohol helps residual water evaporate as air races through the channel. Issues with drying have been reported in many studies. Aumeran et al reported on a duodenoscope-associated outbreak with extended-spectrum beta lactamase-producing Klebsiella pneumoniae and a reprocessing audit revealed endoscopes were not fully dried before storage [23]. A recent systematic review highlights the importance of strict adherence to drying guidelines to make drying procedures more standardized and automated [24].

It is known that safe reprocessing is the key to patient safety and that non-compliance with guidelines and deviations from standardized protocols lead to reprocessing faults with the possibility of patient-to-patient transmission. Recent outbreaks of multidrug-resistant bacteria show how narrow the margin of safety is without compliance with protocols [25]. However, most endoscope reprocessing lapses are never reported or associated infections are not recognized, and if an outbreak has not been contained, it may not be reported. Moreover, it is very likely that outbreaks may not be acknowledged because they involve commensal bacteria of the gastrointestinal tract [26].

A statistically significant difference between the answers to the SIED-ANOTE checklist of indicators submitted to the inspection team before the site visit and the situation found on- site has also been noted in our study. This casts serious doubt on the utility of frequent surveys relying on participant self-reports. Participants may overestimate their own expertise or knowledge or may wish to limit embarrassment or answer in ways that make them "look better" [27].

It is relevant that the improvements obtained after accreditation are not maintained in subsequent years. In fact, in 13 centers that had positively complied with all SIED-ANOTE requirements in the first on-site visit, 2 years later, 23 unsatisfied standards were still detected. The findings especially concern cleansing and the incorrect or non-performance of microbiological tests. It should be noted that in our study, three centers did not have any findings in either the first or the second site visit for renewal of accreditation.

Similar problems of non-compliance with guidelines and standards have been found in other fields of medicine. One study reported that in cardiology, the maximum associated effectiveness of accreditation may be limited over time, supporting the rationale of reaccreditation, which may promote a longlasting effect and help limit subsequent decline in associated benefits [28].

Another study demonstrated that subsequent accreditation surveys significantly reduce variation in quality performance, which correlates with higher reliability [29].

The results of this study need to be interpreted in the context of potential limitations. The generalizability of this study may be limited due to the self-selection of sites entering the SIED-ANOTE accreditation program. This may limit our action and only represent the tip of the iceberg. Moreover, we only visited centers of reference. However, the endoscopic reports, processes, and different reprocessing steps were directly assessed by an experienced multidisciplinary group. 


\section{Conclusions}

In conclusion, our study demonstrates shortcomings in many reprocessing phases in endoscopy centers with a high volume of endoscopic exams subject to the SIED-ANOTE voluntary accreditation program. It also highlights the need for continuous surveillance, with periodic on-site visits, to maintain high safety standards.

\section{Acknowlegements}

The study received unconditional support from the Italian Society for Digestive Endoscopy (SIED). SIED had no influence on the study design, conduct, analysis, or the final manuscript. The authors thank the SIED President Luigi Pasquale, the Central SIED Committee, and ANOTE for their support. They also thank all endoscopists (Baldassarre G, Bertani H, Boarino V, Coppola F, Currò E, Fasoli R, Germanà B, lerfone N, lori V, Lauri A, Marraccini B, Rando G, Santucci R, Tringali A) and nurses (Berenato E, Di Franco A, Gaggiotti M, Giaquinto A, lori G, Marziali $B$, Minenna A, Valdinoci M, Vecchi E, Zamboni $G$ ) engaged in site visits to Italian endoscopy centers. The authors are also grateful to Kiwacermet for technical assistance.

\section{Competing interests}

The authors declare that they have no conflict of interest.

\section{References}

[1] Spaulding EH, Kemmons EX. Chemical disinfection. Am J Nurs 1958; 58: $1238-1212$

[2] Nelson DB, Jarvis WR, Rutala WA et al. Multi-society guideline for reprocessing flexible gastrointestinal endoscopes. Infect Control Hosp Epidemiol 2003; 24: 532-537

[3] Gillespie TG, Hogg L, Budge E et al. Mycobacterium Cholonae isolated from rinse water within an endoscope washer-disinfector. J Hops Infect 2000; 45: 332-334

[4] FDA executive summary: effective reprocessing of endoscopes used in Endoscopic Retrograde Cholangio-Pancreatopraphy(ERCP) procedure 2015. http://www.fda.gov/downloads/UCM445592.pdf

[5] FDA Endoscopic Retrograde Cholangiopancreatography (ERCP) duodenoscope. FDA safety communication. Design may impede effective cleaning. 2015: http://www.fda.gov/safety/UCM434922.htm

[6] Larsen S, Kalloo A, Hutfless S. The hidden cost of colonoscopy including cost of reprocessing and infection rate: the implication for disposable colonoscopies. Gut 2020; 69: 197-200

[7] Wang P, Xu T, Ngamvengphong $S$ et al. Rates of infection after colonoscopy and oesophago-gastroduodenoscopy in ambulatory surgery centres in the USA. Gut 2018; 67: 1626-1636

[8] Tavernise S. Deadly CRE germs linked to hard-to-clean medical scopes. The New York Times 19.02 2015: A14

[9] Beilenhoff U, Biering H, Blum R et al. Reprocessing of flexible endoscopes and endoscopic accessories used in gastrointestinal endoscopy: position statement of the European Society of Gastrointestinal endoscopy (ESGE) and European Society of Gastroenterology Nurses Associates (ESGENA)-Update 2018. Endoscopy 2018; 50: 1205-1234
[10] Dunkley I, Griffiths H, Follows R et al. UK consensus on non-medical staffing required to deliver safe, quality-assured care for patients undergoing gastrointestinal endoscopy. Frontline Gastroenterol 2019; 10: $24-34$

[11] British Society of Gastrointestinal Guidelines for Decontamination of equipment for gastrointestinal endoscopy 2016. http://www.Bsg.org. uk/clinical-guideline/general/guidelines-for-decontamination-ofequipment-for-gastrointestinal-endoscopy-html

[12] Spinzi G, Milano A, Brosolo P et al. The Italian Society for Digestive Endoscopy (SIED) Accreditation and quality improving project based on international standards. Endosc Int Open 2020; 8: E338-E345

[13] Linee Guida pulizia e disinfezione in Endoscopia-update 2011. www. anoteanigea.it/linee-guida-public/linee-guida-pulizia-e-disinfezionein-endoscopia

[14] Peterson BT, Cohen JC, Hambrick RD et al. Multisociety guideline on reprocessing flexible gastrointestinal endoscopes: 2016 update. Gastrointest Endosc 2017; 85: 282-294

[15] Speer T, Alfa M, Cowen A et al. Endoscope disinfection update: a guide to resource-sensitive reprocessing. April 2019 WGO. http:// guide.medlive.cn

[16] Thaler AM, Muthusamy VR, Sederat A et al. Duodenoscope reprocessing practice patterns in US endoscopy centers: a survey study. Gastrointest Endosc 2018; 88: 612-619

[17] Kenters N, Tartari E, Hopman J et al. Worldwide practices on flexible endoscope reprocessing. Antimicrob Resist Infect Control 2018; 7: 153

[18] McCafferty CE, Aghajani M], Abi-Hanna D et al. An update on gastrointestinal endoscopy-associated infections and their contributing factors. Ann Clin Microbiol Antimicrob 2018; 17: 36

[19] Cheung DY, Jang BIK, Kim SW. Multidisciplinary and multi-society practice guidelines on reprocessing flexible gastrointestinal endoscopes and endoscopic accessories. Clin Endosc 2020; 53: 276-285

[20] Park JBG, Yang JN, Lim Y] et al. Survey of endoscope reprocessing in Korea. Clin Endosc 2015; 48: 39-47

[21] Moses FM, Lee JS. Current gastrointestinal endoscope disinfection and QA practices. Dig Dis Sci 2004; 49: 1791-1797

[22] Soares JB, Goncalves R, Banhudo A et al. Reprocessing practice in digestive endoscopy units of district hospitals: results of a Portuguese National Survey. Eur J Gastroenterol Hepatol 2011; 23: 1064-1068

[23] Aumeran C, Poincloux L, Souweine B et al. Multidrug-resistant Klebsiella pneumoniae outbreak after endoscopic retrograde cholangiopancreatography. Endoscopy 2010; 42: 895-899

[24] Tian H, Sun J, Guo S et al. The effectiveness of drying on residual droplets, microorganisms, and biofilms in gastrointestinal endoscope reprocessing: a systematic review. Gastroenterol Res Pract 2021; 2021: 6615357

[25] Jung M, Beilenhoff U. Hygiene: the looming Achilles heel in endoscopy. Visc Med 2016; 31: 21-28

[26] Gastmeier P, Vonberg RP. Klebsiella spp. in endoscopy-associated infections: we may only be seeing the tip of the iceberg. Infection 2014; 42: $15-21$

[27] Colbert CY, Diaz-Guzman E, Meyers JD et al. How to interpret surveys in medical research: a practical approach. Cleve Clin J Med 2013; 80: 423-425

[28] Fan F, Li Y, Zhang Y et al. Chest pain center accreditation is associated with improved in-hospital outcomes of acute myocardial infarction patients in China: findings from the CCC-ACS project. J Am Heart Assoc 2019; 8: e013384

[29] Devkaran S, O'Farrell PN, Ellahham S et al. Impact of repeated hospital accreditation surveys on quality and reliability, an 8-year interrupted time series analysis. BM] Open 2019; 9: e024514 